Astrophys. J. 464, 114(1996)

\title{
Statistics of Extreme Gravitational Lensing Events. I. The Zero Shear Case
}

\author{
Yun Wang \\ NASA/Fermilab Astrophysics Center \\ Fermi National Accelerator Laboratory, Batavia, IL 60510-0500 \\ email: ywang@fnas12.fnal.gov \\ Edwin L. Turner \\ Princeton University Observatory \\ Peyton Hall, Princeton, NJ 08544 \\ email: elt@astro.princeton.edu
}

\begin{abstract}
For a given source and lens pair, there is a thin on-axis tube-like volume behind the lens in which the radiation flux from the source is greatly increased due to gravitational lensing. Any objects (such as dust grains) which pass through such a thin tube will experience strong bursts of radiation, i.e., Extreme Gravitational Lensing Events (EGLE). We study the physics and statistics of EGLE for the case in which finite source size is more important than shear. One of the several possible significant astrophysical effects is investigated with an illustrative calculation.
\end{abstract}




\section{Introduction}

Gravitational lensing has been studied almost exclusively in the context of direct observation of lensed sources from the Earth or a spacecraft (Blandford and Narayan 1992, Schneider et al. 1992). However, every astrophysical object receives the light from sources lensed by intervening massive objects. The most powerful gravitational lensing events occur when the source, lens, and a target object are nearly on-axis. Such events are extreme in magnification, and rare in occurrence for any given target. By considering arbitrarily located targets, we can study the statistics of Extreme Gravitational Lensing Events (EGLE). EGLE can have a significant effect on certain objects, especially fragile components of the interstellar medium, such as molecules or dust grains.

For a pair of source and lens, an EGLE occurs when a moving target object crosses the source-lens line behind the lens. The maximum magnification of the source seen by the target can be extremely large, limited only by the source size and the shear on the lens. As the target moves away from the line connecting the source and the lens, the magnification of the source decreases. The duration of an EGLE depends on the velocity of the target and the size of the high flux region. A slowly moving target in the neighborhood of a pair of small-size source and slightly-sheared lens can experience a strong burst of radiation due to the lensing of the source, which may be sufficient to affect the target's properties.

If the target moves a distance $d$ away from the line connecting the source and the lens, it is equivalent to the source moving an angular distance of $y$ from the optical axis (the line connecting the lens and the target). Measuring $y$ in units of the angular Einstein radius, we have

$$
y \simeq\left(\frac{D_{\mathrm{ds}}}{D_{\mathrm{d}}}\right) \frac{d}{D_{\mathrm{s}} \theta_{\mathrm{E}}},
$$

where $D_{\mathrm{ds}}, D_{\mathrm{s}}$, and $D_{\mathrm{d}}$ are angular diameter distances between the lens and source, target

and source, target and lens respectively. $\theta_{\mathrm{E}}=\sqrt{2 R_{\mathrm{S}} D_{\mathrm{ds}} /\left(D_{\mathrm{d}} D_{\mathrm{s}}\right)}$ is the angular Einstein 
radius. $R_{\mathrm{S}}=2 G M$ is the Schwarzschild radius of the lens with mass $M$. Eq.(过) is the small angle approximation, valid for $d \ll D_{\mathrm{d}}$.

We can write $\theta_{\mathrm{E}}$ as

$$
\theta_{\mathrm{E}}=10^{-6} \times \sqrt{\left(\frac{M}{5 \times 10^{6} M_{\odot}}\right)\left(\frac{1 \mathrm{Mpc}}{D_{\mathrm{d}}}\right) \frac{D_{\mathrm{ds}}}{D_{\mathrm{s}}}} .
$$

The dimensionless radius of a source with physical radius $\rho$ is defined as

$$
R \equiv \frac{\rho}{D_{\mathrm{s}} \theta_{\mathrm{E}}}=\left(\frac{\rho}{1 \mathrm{pc}}\right)\left(\frac{10^{-6}}{\theta_{\mathrm{E}}}\right)\left(\frac{1 \mathrm{Mpc}}{D_{\mathrm{s}}}\right) .
$$

For a given pair of lens and source, the shear $\gamma$ on the lens due to other lensing objects near the line-of-sight is the same order of magnitude as the optical depth for microlensing, $\tau$, the probability that the source is lensed. We find

$$
\gamma=\sqrt{2} \zeta\left(\frac{3}{2}\right) \tau \simeq 3.7 \tau
$$

$\zeta(x)$ is the Riemann zeta function. Not surprisingly, the statistics of EGLE is much more complicated for sheared lenses than for isolated lenses $(\gamma=0)$. Since $\tau=\Omega_{\mathrm{L}} z_{\mathrm{Q}}^{2} / 4\left(\Omega_{\mathrm{L}}\right.$ is the critical density fraction in lenses and $z_{\mathrm{Q}}$ is the source redshift), the shear $\gamma$ is probably small in the low redshift Universe. (Turner 1980, Turner et al. 1984)

The lens model for a perturbed Schwarzschild lens (i.e., point mass lens with shear) has been investigated by Chang and Refsdal $(1979,1984)$ and Subramanian and Chitre (1985). For $\gamma \ll 1$, the caustic is an astroid shaped curve with four cusps. The magnification of extended sources by a perturbed Schwarzschild lens has been studied by Chang (1984), Schneider and Weiss (1987). For a finite source with dimensionless radius $R$ which crosses the optical axis, Figure 1 shows the typical lightcurves for $\gamma=0$ (solid line), $\gamma=R / 2$ (dotted line), $\gamma=R$ (short dashed line), $\gamma=5 R$ (long dashed line), and $\gamma=10 R$ (dot-dashed line) respectively; Figure 2 shows the corresponding cross-sections of magnification. Clearly, 
shear is not important for $\gamma<R \ll 1$. Note that the peaks in the lightcurves in Fig.1 correspond to caustic crossing.

In this paper, we study the statistics of EGLE for sources with small dimensionless radius $R$ and isolated lenses $(\gamma=0)$. We generally follow the notation and conventions of Schneider et al. (1992). We will discuss the statistics of EGLE for lenses with small shear $\gamma$ elsewhere (Wang and Turner, in preparation).

\section{Basic statistics}

In this section, we discuss mean and rms magnifications, as well as integrated excess flux (IEF) seen by a target, for finite sources and Schwarzschild lenses (i.e., point mass lens, no shear).

The magnification of a finite source by a Schwarzschild lens has been studied by Bontz (1979) and Schneider (1987). The magnification of a source with dimensionless radius $R$ is given by (Schneider 1987)

$$
\mu_{\mathrm{e}}(y, R) \simeq \begin{cases}\frac{1}{R} \zeta\left(\frac{y}{R}\right) & \text { for } y \lesssim 5 R \\ \mu_{\mathrm{p}}(y)=\frac{\left(y^{2}+2\right)}{y \sqrt{y^{2}+4}} & \text { for } y \gtrsim 5 R\end{cases}
$$

where

$$
\zeta(w)=\frac{2}{\pi} \int_{0}^{1} \mathrm{~d} x x \int_{0}^{\pi} \frac{\mathrm{d} \phi}{\sqrt{w^{2}+x^{2}+2 w x \cos \phi}} .
$$

$\zeta(0)=2, \zeta(w>0)$ can be easily integrated numerically.

Using Eq.(11), we can define the half-widths of observables in the source plane. Let us define the half-width of the light curve seen by the target to be $y_{\mathrm{HM}}^{\mathrm{w}}$, the source's distance to the optical axis when its magnification goes down to $1 / 2$ its maximum $\mu_{\max }$. 
It's straightforward to find:

$$
\mu_{\max }=\frac{2}{R}, \quad y_{\mathrm{HM}}^{\mathrm{w}} \equiv y\left(\mu=0.5 \mu_{\max }\right) \simeq 1.145 R
$$

The mean, root mean square, and root variance magnifications are given by

$$
\begin{aligned}
& \langle\mu\rangle\left(y \leq y_{\mathrm{HM}}^{\mathrm{w}}\right) \simeq \frac{1.5583}{R}, \quad \sqrt{\left\langle\mu^{2}\right\rangle}\left(y \leq y_{\mathrm{HM}}^{\mathrm{w}}\right) \simeq \frac{1.588}{R} \\
& \sqrt{\left\langle(\mu-\langle\mu\rangle)^{2}\right\rangle}\left(y \leq y_{\mathrm{HM}}^{\mathrm{w}}\right) \simeq \frac{0.3}{R}
\end{aligned}
$$

We are also interested in the integrated excess flux $F$ seen by a moving target. Let us define $t=0$ to be the moment when the moving target crosses the line connecting the source and lens. For a target moving at constant velocity $v$, its distance from the line connecting the source and lens is $d=v t$. Using Eq.(11), we have

$$
\begin{aligned}
F(t) & \equiv \int_{0}^{t} \mathrm{~d} t\left[\mu_{\mathrm{e}}(t, R)-1\right] \\
& =\frac{D_{\mathrm{d}}}{D_{\mathrm{ds}}} \frac{D_{\mathrm{s}} \theta_{\mathrm{E}}}{v} \int_{0}^{y} \mathrm{~d} y\left[\mu_{\mathrm{e}}(y, R)-1\right] \equiv \frac{D_{\mathrm{d}}}{D_{\mathrm{ds}}} \frac{D_{\mathrm{s}} \theta_{\mathrm{E}}}{v} \bar{F}(y) .
\end{aligned}
$$

Using Eqs.(5), we find

$$
\bar{F}_{\text {total }} \equiv \int_{0}^{\infty} \mathrm{d} y\left[\mu_{\mathrm{e}}(y, R)-1\right] \simeq 1.27-\ln R
$$

for $R \lesssim 0.05$. We define the half-width of the integrated excess flux (IEF) to be $y_{\mathrm{IEF}}^{\mathrm{w}}$, the source's distance from the optical axis when the IEF seen by the target is half the total IEF, i.e., $\bar{F}\left(y_{\text {IEF }}^{\mathrm{w}}\right)=\bar{F}_{\text {total }} / 2$. We find

$$
y_{\mathrm{IEF}}^{\mathrm{w}}(R) \simeq 0.287 \sqrt{R}
$$

The corresponding mean, root mean square, and root variance magnifications are

$$
\begin{aligned}
& \langle\mu\rangle\left(y \leq y_{\mathrm{IEF}}^{\mathrm{w}}\right) \simeq \frac{6.97}{\sqrt{R}}, \quad \sqrt{\left\langle\mu^{2}\right\rangle}\left(y \leq y_{\mathrm{IEF}}^{\mathrm{w}}\right) \simeq \sqrt{\frac{24.28}{R} \ln \left(\frac{1.466}{\sqrt{R}}\right)} \\
& \sqrt{\left\langle(\mu-\langle\mu\rangle)^{2}\right\rangle}\left(y \leq y_{\mathrm{IEF}}^{\mathrm{w}}\right) \simeq \frac{4.93}{\sqrt{R}} \sqrt{\ln \left(\frac{0.2}{\sqrt{R}}\right)} .
\end{aligned}
$$


It is useful to consider only the $y<1$ regime, the half-width of microlensing events. The corresponding mean, root mean square, and root variance magnifications are

$$
\begin{aligned}
& \langle\mu\rangle(y \leq 1) \simeq 2.236-0.06 R, \quad \sqrt{\left\langle\mu^{2}\right\rangle}(y \leq 1) \simeq \sqrt{2 \ln \left(\frac{7.531}{R}\right)}, \\
& \sqrt{\left\langle(\mu-\langle\mu\rangle)^{2}\right\rangle}(y \leq 1) \simeq \sqrt{2 \ln \left(\frac{0.6182}{R}\right)} .
\end{aligned}
$$

Generally, for $y \gtrsim 5 R$, we have

$$
\begin{aligned}
& \langle\mu\rangle(\leq y) \simeq \frac{\sqrt{y^{2}+4}}{y}-\frac{0.06 R}{y^{2}} \\
& \left\langle\mu^{2}\right\rangle(\leq y) \simeq 1+\frac{2}{y^{2}}\left[\ln \left(\frac{10.2135}{R}\right)-\ln \left(\frac{\sqrt{y^{2}+4}}{y}\right)\right] .
\end{aligned}
$$

For $y \rightarrow \infty,\langle\mu\rangle=1$, as required by flux conservation, and $\left\langle\mu^{2}\right\rangle=1$. However, it is not physical to average over $y$ from 0 to infinity. For a given source, there is a natural cut-off $y_{\max }$ which is given by the source's distance to the nearest lens, i.e., $y_{\max } \sim 1 / \sqrt{\tau}$, where $\tau$ is the optical depth.

\section{EGLE volume statistics for a point source}

Let us consider a point source $\mathrm{S}$ with luminosity $L_{\mathrm{S}}$, being lensed by a lens $\mathrm{L}$ with Schwarzschild radius $R_{\mathrm{S}}$ (mass $M$ ) at a distance $D_{\mathrm{ds}}$. Let $Q$ denote either the magnification or the flux of the source seen by the target. In a narrow tube-shaped volume $V_{\mathrm{SL}}$ behind the lens, which extends from the lens and tapers off to infinity, $Q$ exceeds some value $q$. The cross-section of the tube is

$$
\sigma(q)=\pi d^{2}=\pi\left[\left(\frac{D_{\mathrm{d}}}{D_{\mathrm{ds}}}\right)^{2} D_{\mathrm{s}}^{2} \theta_{\mathrm{E}}^{2}\right] y^{2}(q),
$$

where we have used Eq.(11). $y(q)$ is the source's dimensionless distance from the optical axis when $Q$ equals $q$. Hence

$$
V_{\mathrm{SL}}(q)=\int_{0}^{D_{\mathrm{d}}(q)} \mathrm{d} D_{\mathrm{d}} \sigma(q) .
$$


Summing over $\mathrm{S}$ gives the total volume $V_{\mathrm{L}}$ in which $Q$ exceeds $q$ for a given lens $\mathrm{L}$; further summing over L gives the total volume $V_{\text {tot }}(>q)$. We use $D_{\mathrm{s}}=D_{\mathrm{d}}+D_{\mathrm{ds}}$ for simplicity in our calculations.

Let us consider the volume $V_{\mathrm{SL}}(f)$ behind the lens in which the flux from the source exceeds $f$. In the absence of magnification, the flux from the source is $f_{0}=L_{\mathrm{S}} /\left(4 \pi D_{\mathrm{s}}^{2}\right)$. The magnified flux $f=\mu f_{0}$. Since we are only interested in high magnification events, we use $y(f) \simeq 1 / \mu=f_{0} / f$ in calculating the cross-section $\sigma(f)$ of $V_{\mathrm{SL}}(f)$. We find

$$
\sigma\left(f, D_{\mathrm{d}}\right)=\frac{2 \pi R_{\mathrm{S}}}{D_{\mathrm{ds}}} \cdot \frac{D_{\mathrm{d}}}{D_{\mathrm{s}}^{3}}\left(\frac{L_{\mathrm{S}}}{4 \pi f}\right)^{2} .
$$

$\sigma\left(f, D_{\mathrm{d}}\right)$ is maximum at $D_{\mathrm{d}}=D_{\mathrm{ds}} / 2$. We find

$$
\sigma_{\mathrm{p}}^{\max }(f)=\frac{8 \pi R_{\mathrm{S}}}{27 D_{\mathrm{ds}}^{3}}\left(\frac{L_{\mathrm{S}}}{4 \pi f}\right)^{2} .
$$

We can define $\sigma_{\mathrm{p}}^{\max }(f)$ to be the characteristic cross-section of the high-flux $(>f)$ tube. The lens which is closest to the source has the thickest high-flux tube behind it.

Using Eq.(17), we obtain

$$
V_{\mathrm{SL}}(f) \simeq \frac{\pi R_{\mathrm{S}}}{D_{\mathrm{ds}}^{2}}\left(\frac{L_{\mathrm{S}}}{4 \pi f}\right)^{2}, \quad V_{\mathrm{L}}(f)=4 \pi^{2} n_{\mathrm{S}} R_{\mathrm{S}} D_{\mathrm{c}}\left(\frac{L_{\mathrm{S}}}{4 \pi f}\right)^{2},
$$

where $n_{\mathrm{S}}$ is the number density of sources, and $D_{\mathrm{c}}$ is the size of the system. In realistic astrophysical systems, the spatial distribution of sources, lenses and targets is complex and varied. In this paper we will consider all systems to be spheres of diameter $D_{\mathrm{c}}$ with all three of these populations distributed uniformly and randomly throughout, a simple but reasonably general approximation. In effect, $D_{\mathrm{c}}$ is the maximum distance between a lens and a source.

Let $\mathcal{F}_{\mathrm{L}}(f)$ be the volume fraction of space in which the flux from the source exceeds $f$ due to gravitational lensing. $\mathcal{F}_{\mathrm{L}}(f)$ should be compared with the volume fraction of space 
$\mathcal{F}_{\mathrm{S}}(f)$ in which the flux from the source exceeds $f$ due to being close to the source. We have

$$
\begin{aligned}
& \mathcal{F}_{\mathrm{L}}(f)=n_{\mathrm{L}} V_{\mathrm{L}}(f)=\frac{3}{2} \tau N_{\mathrm{S}}\left(\frac{f}{f_{\text {min }}}\right)^{-2}, \quad \mathcal{F}_{\mathrm{S}}(f)=N_{\mathrm{S}}\left(\frac{f}{f_{\min }}\right)^{-3 / 2}, \\
& \frac{\mathcal{F}_{\mathrm{L}}(f)}{\mathcal{F}_{\mathrm{S}}(f)}=\frac{3}{2} \tau\left(\frac{f}{f_{\text {min }}}\right)^{-1 / 2}
\end{aligned}
$$

where $\tau$ is the optical depth, $N_{\mathrm{S}}$ is the total number of sources, and $f_{\min }=L_{\mathrm{S}} /\left(4 \pi D_{\mathrm{c}}^{2}\right)$. Note that the volume weighted rms flux due to lensing diverges logarithmically.

The average flux from the general population of sources is $n_{\mathrm{S}} D_{\mathrm{c}} L_{\mathrm{S}}$. Let us define relative flux

$$
f^{\prime}=\frac{f}{n_{\mathrm{S}} D_{\mathrm{c}} L_{\mathrm{S}}} .
$$

Let $\mathcal{F}_{\mathrm{L}}\left(f^{\prime}\right)$ and $\mathcal{F}_{\mathrm{S}}\left(f^{\prime}\right)$ be the volume fractions of space in which the relative flux from the source exceeds $f^{\prime}$ due to lensing and due to being close to the source respectively. We have

$$
\begin{aligned}
& \mathcal{F}_{\mathrm{L}}\left(f^{\prime}, \rho=0\right)=n_{\mathrm{L}} V_{\mathrm{L}}\left(f^{\prime}, \rho=0\right)=\frac{\tau}{6 N_{\mathrm{S}} f^{\prime 2}}, \\
& \frac{\mathcal{F}_{\mathrm{L}}\left(f^{\prime}, \rho=0\right)}{\mathcal{F}_{\mathrm{S}}\left(f^{\prime}\right)}=\frac{\tau}{2}\left(\frac{3}{N_{\mathrm{S}}}\right)^{1 / 2} f^{\prime-1 / 2} .
\end{aligned}
$$

\section{EGLE volume statistics for a finite source}

Now let us consider a source $\mathrm{S}$ with physical radius $\rho$ and luminosity $L_{\mathrm{S}}$, being lensed by a lens $\mathrm{L}$ with Schwarzschild radius $R_{\mathrm{S}}$ (mass $M$ ) at a distance $D_{\mathrm{ds}}$. The tube-shaped volume $V_{\mathrm{SL}}(f, \rho)$ behind the lens in which the flux from the source exceeds $f$ has finite length $D_{\mathrm{d}}^{\mathrm{m}}(f, \rho)$, because of the finite size of the source.

For a finite source with dimensionless radius $R, \mu_{\max }=2 / R$. Let us define a parameter $\alpha(f)$ which measures the maximum magnification of the source relative to the flux $f$,

$$
\alpha \equiv \frac{8 R_{\mathrm{S}} D_{\mathrm{c}}}{\rho^{2}}\left(\frac{L_{\mathrm{S}}}{4 \pi D_{\mathrm{c}}^{2} f}\right)^{2} .
$$


The tube volume $V_{\mathrm{L}}(f, \rho)$ has the cross-section $\sigma\left(f, \rho, D_{\mathrm{d}}\right)$ which vanishes at $D_{\mathrm{d}}=0$, $D_{\mathrm{d}}^{\mathrm{m}}$. To calculate the cross-section $\sigma(f, \rho)$, we need to know $y(f)$ [see Eq.(15)], which can be found by inverting $\mu(y)=f / f_{0}$ numerically. $\sigma(f, \rho)$ can be written as

$$
\sigma\left(f, \rho, D_{\mathrm{d}}\right)=\pi \rho^{2} \times d^{2} / \rho^{2}=\pi \rho^{2} \bar{\sigma}\left(\alpha, D_{\mathrm{d}}\right)
$$

for given $D_{\mathrm{ds}}$. Figure 3 shows the cross-section $\sigma\left(f, \rho, D_{\mathrm{d}}\right)$ with $\alpha(f)=4$, for $D_{\mathrm{ds}}=0.2 D_{\mathrm{c}}$ (solid line), $0.5 D_{\mathrm{c}}$ (long dashed line). The lens which is closest to the source has the thickest tube of high flux behind it, as in the point source case.

To simplify the calculation for the volume fractions, let us approximate Eq.(5) with

$$
\mu_{\mathrm{e}}(y, R) \simeq \begin{cases}\mu_{\mathrm{p}}(y) & \text { for } \mu<\mu_{\max } \\ \mu_{\max } & \text { elsewhere }\end{cases}
$$

where $\mu_{\mathrm{p}}(y)$ is the point source magnification, and $\mu_{\max }=2 / R$. Eq.(25) is reasonably good for $\mu \lesssim 1 / R$. Figure 3 shows the approximate cross-sections obtained by using Eq.(25) for $D_{\mathrm{ds}}=0.2 D_{\mathrm{c}}$ (short dashed line), $0.5 D_{\mathrm{c}}$ (dot-dashed line). The dotted lines indicate $D_{\mathrm{d}}^{\mathrm{m}}$. This approximate cross-section always under-estimates the true cross-section; the difference increases with decreasing $\alpha(f)$ (large $\rho$ or $f$ ), but it is negligible for our purposes.

Now let us derive the length of the tube-volume $V_{\mathrm{SL}}(f, \rho)$. Note that $f=\mu f_{0} \leq \mu_{\max } f_{0}$. Let $f=\mu_{\max } f_{0}$ at $D_{\mathrm{d}}(f)=D_{\mathrm{d}}^{\mathrm{m}}(f)$, i.e., the flux is equal to $f$ on the line SL connecting the source and lens. For given $D_{\mathrm{d}}$, the flux decreases away from line SL, hence the volume in which the flux exceeds $f$ converges to a point at $D_{\mathrm{d}}(f)=D_{\mathrm{d}}^{\mathrm{m}}(f)$. For $D_{\mathrm{d}}>D_{\mathrm{d}}^{\mathrm{m}}$, the volume in which the flux exceeds $f$ is zero. For a given pair of source and lens, $D_{\mathrm{d}}^{\mathrm{m}}(f)$ gives the length of the tube volume in which the flux exceeds $f$. To find $D_{\mathrm{d}}^{\mathrm{m}}(f)$, we write $f=\mu_{\max } f_{0}$ as

$$
\frac{D_{\mathrm{d}}^{\mathrm{m}}}{D_{\mathrm{c}}}\left(\frac{D_{\mathrm{d}}^{\mathrm{m}}}{D_{\mathrm{c}}}+\frac{D_{\mathrm{ds}}}{D_{\mathrm{c}}}\right)^{3}=\frac{8 R_{\mathrm{S}} D_{\mathrm{ds}}}{\rho^{2}}\left(\frac{L_{\mathrm{S}}}{4 \pi D_{\mathrm{c}}^{2} f}\right)^{2}=\alpha\left(\frac{D_{\mathrm{ds}}}{D_{\mathrm{c}}}\right)
$$


The above equation can be solved analytically for $D_{\mathrm{d}}^{\mathrm{m}}(f)$. Let us define

$$
x \equiv \frac{D_{\mathrm{ds}}}{D_{\mathrm{c}}}, \quad \omega \equiv \frac{x}{\alpha^{1 / 3}} .
$$

We find

$$
\frac{D_{\mathrm{d}}^{\mathrm{m}}(f)}{D_{\mathrm{ds}}}=\frac{1}{2}\left\{\frac{[\omega u(\omega)]^{3 / 2}}{2}+\sqrt{\frac{1}{2}\left[1+[\omega u(\omega)]^{-3 / 2}\right]+u(\omega)}-\frac{3}{2}\right\} \equiv g(\omega),
$$

where

$$
u(\omega)=\frac{3}{4}\left[b(\omega)+\frac{1}{b(\omega)}+1\right]^{-1}, \quad b(\omega)=\frac{3 \omega}{4}\left[\frac{1}{2}+\sqrt{\frac{1}{4}+\left(\frac{4}{3 \omega}\right)^{3}}\right]^{2 / 3}
$$

$g(\omega)=D_{\mathrm{d}}^{\mathrm{m}}(f) / D_{\mathrm{ds}}$ is shown in Figure 4. Given the separation between the source and the lens, $x=D_{\mathrm{ds}} / D_{\mathrm{c}}$, the length of the tube volume behind the lens in which the flux exceeds $f$ is given by $D_{\mathrm{d}}^{\mathrm{m}}(f) / D_{\mathrm{c}}=x g(\omega)$, where $\omega=x \alpha^{-1 / 3}$. The tube length is of order $D_{\mathrm{c}}$ for $\alpha$ of order 1 .

The function $g(\omega)$ has the following asymptotic behavior:

$$
g(\omega)= \begin{cases}\omega^{-3} & \text { for } \omega \gg 1 \\ \omega^{-3 / 4} & \text { for } \omega \ll 1\end{cases}
$$

Thus we have

$$
\frac{D_{\mathrm{d}}^{\mathrm{m}}(f)}{D_{\mathrm{c}}}= \begin{cases}\alpha(f) / x^{2} & \text { for } \alpha^{1 / 3} \ll x \\ {[\alpha(f) x]^{1 / 4}} & \text { for } \alpha^{1 / 3} \gg x\end{cases}
$$

Substitution of Eq.(28) into Eq.(16) gives $V_{\mathrm{SL}}(f, \rho)$. Using Eq.(25) and $\mu_{\mathrm{p}}(y) \simeq 1 / y$ (for high magnification events) in computing $\sigma(f, \rho)$, we find

$$
V_{\mathrm{L}}(f, \rho)=4 \pi^{2} n_{\mathrm{S}} R_{\mathrm{S}} D_{\mathrm{c}}\left(\frac{L_{\mathrm{S}}}{4 \pi f}\right)^{2} I(\alpha)
$$

where

$$
I(\alpha)=\alpha^{1 / 3} \int_{0}^{\alpha^{-1 / 3}} \frac{\mathrm{d} \omega}{[1+1 / g(\omega)]^{2}} .
$$


For high flux, $\alpha(f) \ll 1, I(\alpha)=0.3583 \alpha^{1 / 3}$. Note that $I(\alpha)=V_{\mathrm{L}}(f, \rho) / V_{\mathrm{L}}(f, \rho=0)$. In the point source limit, $\alpha \gg 1, I(\alpha)=1$. We show $I(\alpha)$ in Figure 5 .

Given $I(\alpha)=A \alpha^{\beta}$, with $\mathcal{F}_{\mathrm{L}}(f)$ and $\mathcal{F}_{\mathrm{S}}(f)$ denoting the volume fractions of space in which the flux from the source exceeds $f$ due to lensing and due to being close to the source respectively, we find

$$
\begin{aligned}
& \mathcal{F}_{\mathrm{L}}(f, \rho)=\frac{A}{4 \cdot 2^{\beta} \pi^{2 \beta}} n_{\mathrm{S}} n_{\mathrm{L}} R_{\mathrm{S}}^{1+\beta} L_{\mathrm{S}}^{2+2 \beta} f^{-(2+2 \beta)} D_{\mathrm{c}}^{1-3 \beta} \rho^{-2 \beta} \\
& \frac{\mathcal{F}_{\mathrm{L}}(f, \rho)}{\mathcal{F}_{\mathrm{S}}(f)}=\frac{3 \pi^{1 / 2-2 \beta} A}{2^{1+\beta}} n_{\mathrm{L}} R_{\mathrm{S}}^{1+\beta} L_{\mathrm{S}}^{1 / 2+2 \beta} f^{-(1 / 2+2 \beta)} D_{\mathrm{c}}^{1-3 \beta} \rho^{-2 \beta}
\end{aligned}
$$

\section{Possible astrophysical effects}

For a population of sources (with number density $n_{\mathrm{S}}$ ) lensed by a population of lenses (with number density $n_{\mathrm{L}}$ ), the physical picture for EGLE is a complex network of thin high-flux tubes, at each knot sits a lens, and each tube line points away from a source. In other words, a given lens has one high-flux tube coming out of it because of each source, and a given source induces one high-flux tube behind each lens.

To roughly survey possible astrophysical effects of EGLE, we construct tables of possible source and lens populations. Table 1 lists a few types of small sources with high luminosity. Note that the space density $n_{\mathrm{S}}$ associated with transient sources such as $\gamma$-ray bursts and supernovae includes the finite lifetime factor; in other words, it is the density of sources shining at a particular moment. Table 2 gives two possible lens populations. Again, we have assumed that the sources and the lenses are mixed and both are distributed uniformly in a volume with size $D_{c}$. Here we only consider the volume fractions of space occupied by the high-flux EGLE tubes (which are determined by the sources and lenses only); the results derived in this Section apply to arbitrary targets uniformly distributed in the same volume with the sources and the lenses. 
In the context of EGLE, the relevant dimensional physical quantities are: lens mass $M$, size of the lens-source distribution $D_{\mathrm{c}}$, source size $\rho$, source luminosity $L_{\mathrm{S}}$, minimum lensed flux from the source $f$. All these collapse into a single dimensionless parameter $\alpha(f)$ [see Eq.(23)], which measures the maximum magnification of the source relative to the flux $f$. In astrophysical units, we write

$$
\begin{aligned}
\log (\alpha)=-5.62+0.8 & m_{\text {bol }}+2 \log \left(\frac{L_{\mathrm{S}}}{L_{\odot}}\right)+\log \left(\frac{M}{M_{\odot}}\right)-2 \log \left(\frac{\rho}{R_{\odot}}\right) \\
& -3 \log \left(\frac{D_{\mathrm{c}}}{1 \mathrm{kpc}}\right)
\end{aligned}
$$

where the minimum flux $f$ is measured by $m_{\text {bol }}$. Table 1 shows $\log (\alpha)-0.8 m_{\text {bol }}$ for the listed sources with $M=M_{\odot}$, i.e., for lensing by stars.

Let us first consider the physical dimensions of a high-flux tube behind a lens, in which the flux exceeds $f$. Using Eq.(28), we find

$$
\log \left(\frac{D_{\mathrm{d}}^{\mathrm{m}}}{\mathrm{cm}}\right)=21.49+\log \left(\frac{D_{\mathrm{ds}}}{\mathrm{kpc}}\right)+\log g(\omega),
$$

where $\omega=\left(D_{\mathrm{ds}} / D_{\mathrm{c}}\right) \alpha^{-1 / 3} \cdot g(\omega)$ is given by Eq.(28) and shown in Fig.4. For $\alpha \ll 1, \omega \gg 1$ at a given $D_{\mathrm{ds}}, g(\omega) \simeq \omega^{-3}$; i.e., the length of the high-flux tube decreases sharply for small $\alpha$. In Fig.6, we show $D_{\mathrm{d}}^{\mathrm{m}}$ versus $m_{\text {bol }}$ for lensing by stars, the sources are $\gamma$-ray bursts (solid line), QSO (X-ray) (dotted line), QSO (UV-opt) (short dashed line), Galactic supernovae (long dashed line), neutron stars (dot-short dashed line), hot O stars (dot-long dashed line), and hot B stars (short dash-long dashed line) respectively. Note that we have taken $\gamma$-ray bursts to be cosmological in origin (Mao and Paczynski 1992); models which attribute them to local sources would predict smaller EGLE effects.

Since the cross-section of the high-flux tube for a finite source does not deviate significantly from that of a point source [see Fig.3], we define the characteristic radius $a$ of the high-flux tube to correspond to the maximum cross-section (along the optical axis) of 
the high-flux tube for a point source [see Eq.(18)]. We find

$$
\log \left(\frac{a}{\mathrm{~cm}}\right)=7.38+0.4 m_{\mathrm{bol}}+\log \left(\frac{L_{\mathrm{S}}}{L_{\odot}}\right)+\frac{1}{2} \log \left(\frac{M}{M_{\odot}}\right)-\frac{3}{2} \log \left(\frac{D_{\mathrm{ds}}}{\mathrm{kpc}}\right) .
$$

In Table 1 , we show $\log a-0.4 m_{\text {bol }}$ for the listed sources lensed by stars, with $M=M_{\odot}$ and $D_{\mathrm{ds}}=D_{\mathrm{c}} / 2$.

Now we compute the volume fractions of space occupied by the high-flux tubes, for the sources and lenses in Tables 1 and 2. Let $\mathcal{F}_{\mathrm{L}}(f)$ and $\mathcal{F}_{\mathrm{S}}(f)$ denote the total volume fractions of space in which the flux from the source exceeds $f$ due to gravitational lensing and due to being close to the source respectively. A high-flux tube, in which the flux from the source exceeds $f$, has a bolometric magnitude less than $m_{\text {bol }}$. We find

$$
\begin{aligned}
\log \mathcal{F}_{\mathrm{L}}=-8 & +\log I(\alpha)+0.8 m_{\mathrm{bol}}+2 \log \left(\frac{L_{\mathrm{S}}}{L_{\odot}}\right)+\log \left(\frac{M}{M_{\odot}}\right) \\
& +\log \left(\frac{D_{\mathrm{c}}}{1 \mathrm{kpc}}\right)+\log \left(\frac{n_{\mathrm{S}}}{1 \mathrm{pc}^{-3}}\right)+\log \left(\frac{n_{\mathrm{L}}}{1 \mathrm{pc}^{-3}}\right) \\
\log \left(\frac{\mathcal{F}_{\mathrm{L}}}{\mathcal{F}_{\mathrm{S}}}=-\right. & 8.86+\log I(\alpha)+0.2 m_{\mathrm{bol}}+\frac{1}{2} \log \left(\frac{L_{\mathrm{S}}}{L_{\odot}}\right)+\log \left(\frac{M_{\mathrm{L}}}{M_{\odot}}\right) \\
& +\log \left(\frac{D_{\mathrm{c}}}{1 \mathrm{kpc}}\right)+\log \left(\frac{n_{\mathrm{L}}}{1 \mathrm{pc}^{-3}}\right),
\end{aligned}
$$

where $I(\alpha)$ is the reduction factor due to the finite source size [see Eq.(33) and Fig.5.].

In Fig.7(a), we plot $\log \left(\mathcal{F}_{\mathrm{L}} / \mathcal{F}_{\mathrm{S}}\right)$ for lensing by stars, for the same sources as in Fig.6

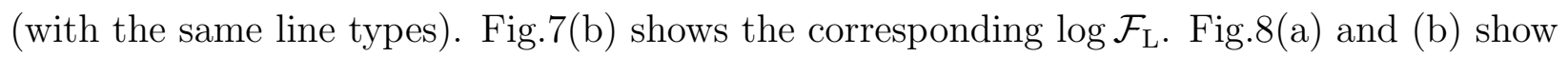
$\log \left(\mathcal{F}_{\mathrm{L}} / \mathcal{F}_{\mathrm{S}}\right)$ and $\log \left(\mathcal{F}_{\mathrm{L}}\right)$ for lensing by giant black holes for the same cosmological sources as in Fig.6 (with the same line types). Only rough order of magnitude properties are used for the sources and lenses in Figs.6-8. The largest effect comes from $\gamma$-ray bursts lensed by stars in our simple model (zero shear).

We note that although the high-flux volume fractions due to lensing are small, the corresponding absolute volumes can be large. Further, since materials move across the 
high flux tubes constantly, the fraction of material affected by EGLE is much higher than the static volume fractions. Finally, recall that we are neglecting shear, which might be important in some of the cases enumerated here.

\section{An example: destruction of dust grains in globular clusters}

As a specific example, we consider the destruction of dust grains in the high-flux tubes produced by EGLE. Let us consider a system of physical size $D_{\mathrm{c}}$, with $N_{\mathrm{S}}$ bright sources, lensed by $N_{\mathrm{L}}$ lenses with mass $M$. The high-flux regions associated with being close to a source consist of $N_{\mathrm{S}}$ small spheres enclosing the sources, while the high-flux regions associated with EGLE consist of thin tubes stretching out from the $N_{\mathrm{L}}$ lenses distributed all over the system.

For a given dust grain in this system, the instantaneous probability that an EGLE produces flux greater than $f$ is given by $\mathcal{F}_{\mathrm{L}}(f)$, the volume fraction of space in tubes with flux greater than $f$. The typical duration of this EGLE, $\Delta t$, is given by $2 a / v$, where $a$ is the characteristic radius of the high-flux tubes, and $v$ is the typical velocity of the dust grain. The time between such events is given by $t_{f}=\Delta t / \mathcal{F}_{\mathrm{L}}(f)$. For dust grains to be destroyed by EGLE, the following two conditions are sufficient: (1) One EGLE heats up a dust grain to sufficiently high temperature for a sufficient amount of time to destroy the dust grain; (2) $t_{f}$ is less than the typical lifetime of a dust grain in the absence of EGLE.

We now focus on the specific example of dust grain destruction within a typical globular cluster. In such a cluster, there are approximately 10 bright X-ray/UV sources associated with accreting neutron stars, which are lensed by $10^{6}$ stars. The bolometric luminosity of these sources is thought to be of order their Eddington luminosities. The parameter $\alpha$, which measures the maximum magnification of the source relative to the flux 
$f$ [see Eqs.(23) and (35)], is given by

$$
\begin{aligned}
\log (\alpha)=21.38+0.8 & m_{\text {bol }}(f)+2 \log \left(\frac{L_{\mathrm{S}}}{10^{5} L_{\odot}}\right)+\log \left(\frac{M}{M_{\odot}}\right)-2 \log \left(\frac{\rho}{10^{-4} R_{\odot}}\right) \\
& -3 \log \left(\frac{D_{\mathrm{c}}}{1 \mathrm{pc}}\right)
\end{aligned}
$$

The optical depth for microlensing in this system is $\tau \sim 3 \times 10^{-7}$, which means the shear $\gamma \sim 10^{-6}$ [see Eq.(44)]; The dimensionless radius of the source is $R \sim 5 \times 10^{-6}$. Clearly, shear is not important in this system [see Figs. 1 and 2]. Also, note that even though the lens, source, and target here are all within a few parsecs of each other (separations very small compared to most of the lensing situations currently discussed in the literature), the Einstein ring radius of the lens is still much larger [by roughly 10 times] than the physical size of the star-lens; hence the lens can be safely approximated as "transparent". The formalism developed in the previous sections applies here.

The volume fraction of space occupied by high-flux tubes with bolometric magnitude less than $m_{\text {bol }}$ is

$$
\begin{gathered}
\log \mathcal{F}_{\mathrm{L}}=6.562+\log I(\alpha)+0.8 m_{\mathrm{bol}}+2 \log \left(\frac{L_{\mathrm{S}}}{10^{5} L_{\odot}}\right)+\log \left(\frac{M}{M_{\odot}}\right) \\
-5 \log \left(\frac{D_{\mathrm{c}}}{1 \mathrm{pc}}\right)+\log \left(\frac{N_{\mathrm{S}}}{10}\right)+\log \left(\frac{N_{\mathrm{L}}}{10^{6}}\right)
\end{gathered}
$$

where $I(\alpha)$ is the reduction factor due to the finite source size [see Eq.(33) and Fig.5.]. The length of a high-flux tube is

$$
\log \left(\frac{D_{\mathrm{d}}^{\mathrm{m}}}{\mathrm{pc}}\right)=\log \left(\frac{D_{\mathrm{ds}}}{\mathrm{pc}}\right)+\log g(\omega)
$$

where $\omega=\left(D_{\mathrm{ds}} / D_{\mathrm{c}}\right) \alpha^{-1 / 3} \cdot g(\omega)$ is given by Eq.(28) and shown in Fig.4. Note that if the high-flux tube is longer than the size of the system $D_{\mathrm{c}}$, only the part of the high-flux tube which lies inside the system contributes to the dust grain heating in the system. The characteristic radius $a$ of the high-flux tube is

$$
\log \left(\frac{a}{\mathrm{~cm}}\right)=16.88+0.4 m_{\mathrm{bol}}+\log \left(\frac{L_{\mathrm{S}}}{10^{5} L_{\odot}}\right)+\frac{1}{2} \log \left(\frac{M}{M_{\odot}}\right)-\frac{3}{2} \log \left(\frac{D_{\mathrm{ds}}}{\mathrm{pc}}\right) .
$$


Assuming 100\% efficiency in the absorption of photons by the dust grains (as appropriate in the UV and soft X-ray bands), the relation of the grain temperature $T$ to the photon flux $f$ is given by (Draine and Lee 1984)

$$
f=4\left\langle Q\left(a_{\mathrm{gr}}, T\right)\right\rangle \sigma T^{4}
$$

where $\left\langle Q\left(a_{\mathrm{gr}}, T\right)\right\rangle$ is the Planck-averaged emissivity for a dust grain with radius $a_{\mathrm{gr}}$, and $\sigma$ is the Stefan-Boltzmann constant. Hence we have

$$
m_{\mathrm{bol}}=-10 \log \left(\frac{T\left\langle Q\left(a_{\mathrm{gr}}, T\right)\right\rangle^{1 / 4}}{0.56 \mathrm{~K}}\right) .
$$

For a silicate grain, $\left\langle Q\left(a_{\mathrm{gr}}, T\right)\right\rangle \lesssim 0.4 a_{\mathrm{gr}} / \mu \mathrm{m}$ for $100 \mathrm{~K}<T<1000 \mathrm{~K}$; we use this upper limit on the emissivity below.

For a dust grain moving with velocity $v$, the duration of one EGLE is $\Delta t=2 a / v$. We find

$$
\frac{\Delta t}{\mathrm{sec}}=260.5\left(\frac{T}{300 \mathrm{~K}}\right)^{-4}\left(\frac{a_{\mathrm{gr}}}{0.1 \mu \mathrm{m}}\right)^{-1}\left(\frac{L_{\mathrm{S}}}{10^{5} L_{\odot}}\right)\left(\frac{M}{M_{\odot}}\right)^{1 / 2}\left(\frac{D_{\mathrm{ds}}}{0.5 \mathrm{pc}}\right)^{-3 / 2}\left(\frac{v}{5 \mathrm{~km} / \mathrm{sec}}\right)^{-1} .
$$

In Fig.9, we plot $t_{f}=\Delta t / \mathcal{F}_{\mathrm{L}}(f)$, the mean time between EGLEs, as function of the resulting dust grain temperature for three typical values of the grain radius. The bend at low temperature is due to the cut-off in length of the high-flux tubes longer than $D_{\mathrm{c}}=1 \mathrm{pc}$ (the lower the flux or temperature, the longer the corresponding EGLE tube). A silicate grain with $a_{\text {gr }}$ of order $0.1 \mu \mathrm{m}$ can be heated to over $300 \mathrm{~K}$ for a few minutes, once every $5 \times 10^{7}$ years; this would be sufficient to destroy any icy components of the grain. Smaller silicate grains of, say, $0.01 \mu \mathrm{m}$ radius would be more fragile and would be heated to over $1000 \mathrm{~K}$ for 21 seconds once every $8.5 \times 10^{8}$ years, perhaps sufficient to destroy them. Thus, EGLE may provide a mechanism for explaining the observed absence of dust in globular clusters (Knapp, Gunn and Connolly 1995).

Note that although we used the maximum cross-section of the high-flux tubes in estimating the EGLE durations, we also assumed that a dust grain moves through the 
high-flux tube exactly tangentially; if the dust grain enters the high-flux tube at an angle, the duration of the event is longer. In addition, we used the minimum flux $f$ and temperature $T$ which define the event in our calculations; the peak values during each event will be substantially higher than those at the edge of the tube. Furthermore, the dust grain emissivity is likely lower than the extreme value we adopted. Therefore, our estimate of the EGLE effects on grains in globular clusters is rather conservative.

We have also ignored the interaction of globular cluster EGLE with the gaseous intracluster medium that would presumably have accompanied any putative dust component. Of course, EGLE conserve photons and thus total ionizing events (for example), so we do not expect such dramatic effects as when there are threshold phenomena such as dust grain evaporation, but the spatial redistribution of the photons might still have some subtle consequences. A more accurate and detailed examination of this and other possible EGLE effects is beyond the scope of the present paper.

We thank Bruce Draine for very instructive discussions of possible dust grain effects. We also thank the ApJ Scientific Editor E. L. Wright and the anonymous referee for comments which led to a clearer presentation of our results. Y.W. is supported by the DOE and NASA under Grant NAG5-2788. E.L.T. gratefully acknowledges support from NSF grant AST94-19400. 
Table 1: List of possible EGLE sources. $\log \alpha$ and $\log a$ are computed with $M=M_{\odot}$ and $D_{\mathrm{ds}}=D_{\mathrm{c}} / 2$.

\begin{tabular}{|c|c|c|c|c|c|c|c|}
\hline & & $\rho / R_{\odot}$ & $L_{\mathrm{S}} / L_{\odot}$ & $D_{\mathrm{c}} / \mathrm{kpc}$ & $n_{\mathrm{S}} / \mathrm{pc}^{-3}$ & $\begin{array}{l}\log \alpha \\
-0.8 m_{\mathrm{bol}}\end{array}$ & $\begin{array}{l}\log (a / \mathrm{cm}) \\
-0.4 m_{\mathrm{bol}}\end{array}$ \\
\hline QSO & $\begin{array}{l}\text { X-ray } \\
\text { UV-opt }\end{array}$ & $\begin{array}{l}10^{2} \\
10^{4}\end{array}$ & $\begin{array}{l}10^{12} \\
10^{13}\end{array}$ & $10^{6}$ & $3 \times 10^{-22}$ & $\begin{array}{l}-3.62 \\
-5.62\end{array}$ & $\begin{array}{l}10.83 \\
11.83\end{array}$ \\
\hline \multicolumn{2}{|c|}{$\gamma$-ray bursts } & $10^{-5}-0.1$ & $10^{18-20}$ & $10^{6}$ & $10^{-31}$ & $14.38-26.38$ & $16.83-18.83$ \\
\hline \multicolumn{2}{|c|}{ Galactic supernovae } & $10^{3}$ & $10^{10}$ & 10 & $10^{-14}$ & 5.38 & 16.33 \\
\hline neutron stars & $\begin{array}{l}\text { X-ray } \\
\text { radio }\end{array}$ & $10^{-5}$ & $\begin{array}{l}10^{5} \\
10^{6}\end{array}$ & $10^{-2}-1$ & $10^{-9}$ & $\begin{array}{l}14.38-20.38 \\
16.38-22.38\end{array}$ & $\begin{array}{l}12.83-15.83 \\
13.83-16.83\end{array}$ \\
\hline hot stars & $\operatorname{ars}\left\{\begin{array}{l}O \\
B\end{array}\right.$ & $\begin{array}{l}10 \\
4\end{array}$ & $\begin{array}{l}10^{5} \\
10^{3}\end{array}$ & 1 & $\begin{array}{l}10^{-8} \\
10^{-4}\end{array}$ & $\begin{array}{l}2.38 \\
-0.824\end{array}$ & $\begin{array}{l}12.83 \\
10.83\end{array}$ \\
\hline
\end{tabular}

Note. - $D_{\mathrm{c}}$ is the size of the volume in which the sources, the lenses, and the targets are all uniformly distributed. 
Table 2: List of possible EGLE lenses

\begin{tabular}{|c||c|c|}
\hline \multicolumn{1}{|c||}{} & $M$ & $n_{\mathrm{L}}$ \\
\hline $\begin{array}{l}\text { giant black holes } \\
\text { (cosmological) }\end{array}$ & $10^{6-8} M_{\odot}$ & $10^{3} /(\mathrm{Mpc})^{3}$ \\
\hline \multirow{2}{*}{ stars $\begin{array}{l}\text { (galactic average) } \\
\text { (cosmological average) }\end{array}$} & $0.1-1 M_{\odot}$ & $\begin{array}{l}1.4 /(\mathrm{pc})^{3} \\
10^{11} /(\mathrm{Mpc})^{3}\end{array}$ \\
\hline
\end{tabular}




\section{REFERENCES}

Blandford, R.D. and Narayan, R. (1992), Ann. Rev. Astron. Astrophys., 30, 311.

Bontz, R.J. (1979), ApJ, 233, 402.

Chang, K. and Refsdal, S. (1979), Nature, 282, 561.

Chang, K (1984), Astr. Ap., 130, 157.

Chang, K. and Refsdal, S. (1984), Astr. Ap., 132, 168.

Draine, B.T. and Lee, H.M. (1984), ApJ, 285, 89.

Knapp, G.R., Gunn, J.E. and Connolly, A.J. (1995), preprint POP-614.

Mao, S. and Paczynski, B. (1992), ApJ, 388, L45.

Schneider, P. and Weiss, A. (1987), Astr. Ap., 171, 49.

Schneider, P. (1987), Astr. Ap., 179, 71.

Schneider, P., Ehlers, J., Falco, E.E., (ed. Springer-Verlag, Berlin, 1992), "Gravitational lenses".

Subramanian, K. and Chitre, S.M. (1985), ApJ, 289, 37.

Turner, E. L. (1980), ApJ, 242, L135.

Turner, E. L., Ostriker, J. P., and Gott, J. R. (1984), ApJ, 284, 1.

Wang, Y. and Turner, E. L., in preparation. 
Fig. 1.- Typical lightcurves for shear $\gamma=0$ (solid line), $\gamma=R / 2$ (dotted line), $\gamma=R$ (short dashed line), $\gamma=5 R$ (long dashed line), and $\gamma=10 R$ (dot-dashed line). The dimensionless source size $R \ll 1$. $y$ and $\mu$ are the angular position and the maginification of the source respectively.

Fig. 2.- Cross-sections of magnification for lightcurves in Fig.1 (with the same line types).

Fig. 3.- Cross-section $\sigma\left(f, \rho, D_{\mathrm{d}}\right)$ with $\alpha(f)=4$, for $D_{\mathrm{ds}}=0.2 D_{\mathrm{c}}$ (solid line), $0.5 D_{\mathrm{c}}$ (long dashed line).

Fig. 4.- The scaled length of the high-flux $(>f)$ tube, $g(\omega)=D_{\mathrm{d}}^{\mathrm{m}}(f) / D_{\mathrm{ds}}$, as a function of $\omega=\left(D_{\mathrm{ds}} / D_{\mathrm{c}}\right) \alpha^{-1 / 3}(f)$.

Fig. 5.- $I(\alpha)=V_{\mathrm{L}}(f, \rho) / V_{\mathrm{L}}(f, \rho=0)$, the reduction factor in EGLE volumes due to finite source size.

Fig. 6. - The high-flux $(>f)$ tube's length $D_{\mathrm{d}}^{\mathrm{m}}$ versus $m_{\mathrm{bol}}(f)$ for various sources lensed by stars. The sources are $\gamma$-ray bursts (solid line), QSO (X-ray) (dotted line), QSO (UVopt) (short dashed line), Galactic supernovae (long dashed line), neutron stars (dot-short dashed line), hot O stars (dot-long dashed line), and hot B stars (short dash-long dashed line) respectively.

Fig. 7.- Lensing by stars of the same sources as in Fig.6 (with the same line types). (a) $\log \left(\mathcal{F}_{\mathrm{L}} / \mathcal{F}_{\mathrm{S}}\right) \cdot(\mathrm{b}) \log \mathcal{F}_{\mathrm{L}}$

Fig. 8. - Lensing by giant black holes of the same cosmological sources as in Fig.6 (with the same line types). (a) $\log \left(\mathcal{F}_{\mathrm{L}} / \mathcal{F}_{\mathrm{S}}\right)$. (b) $\log \left(\mathcal{F}_{\mathrm{L}}\right)$.

Fig. 9.- The time between two EGLEs as function of the silicate grain temperature, for X-ray/UV sources lensed by stars in a typical globular cluster. The values of the grain radius are labeled on the curves. 


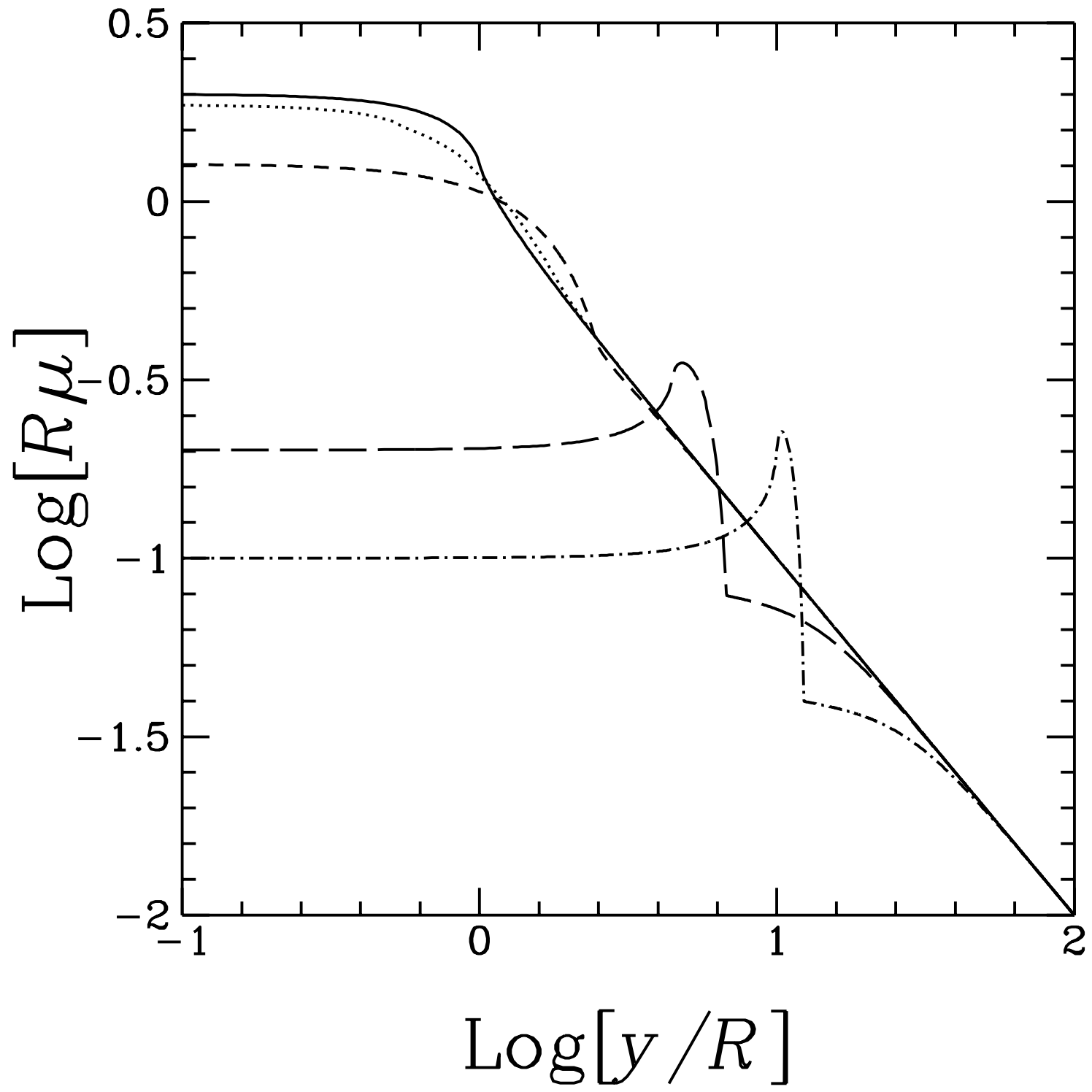

Fig. 1.- Typical lightcurves for shear $\gamma=0$ (solid line), $\gamma=R / 2$ (dotted line), $\gamma=R$ (short dashed line), $\gamma=5 R$ (long dashed line), and $\gamma=10 R$ (dot-dashed line). The dimensionless source size $R \ll 1$. $y$ and $\mu$ are the angular position and the maginification of the source respectively. 


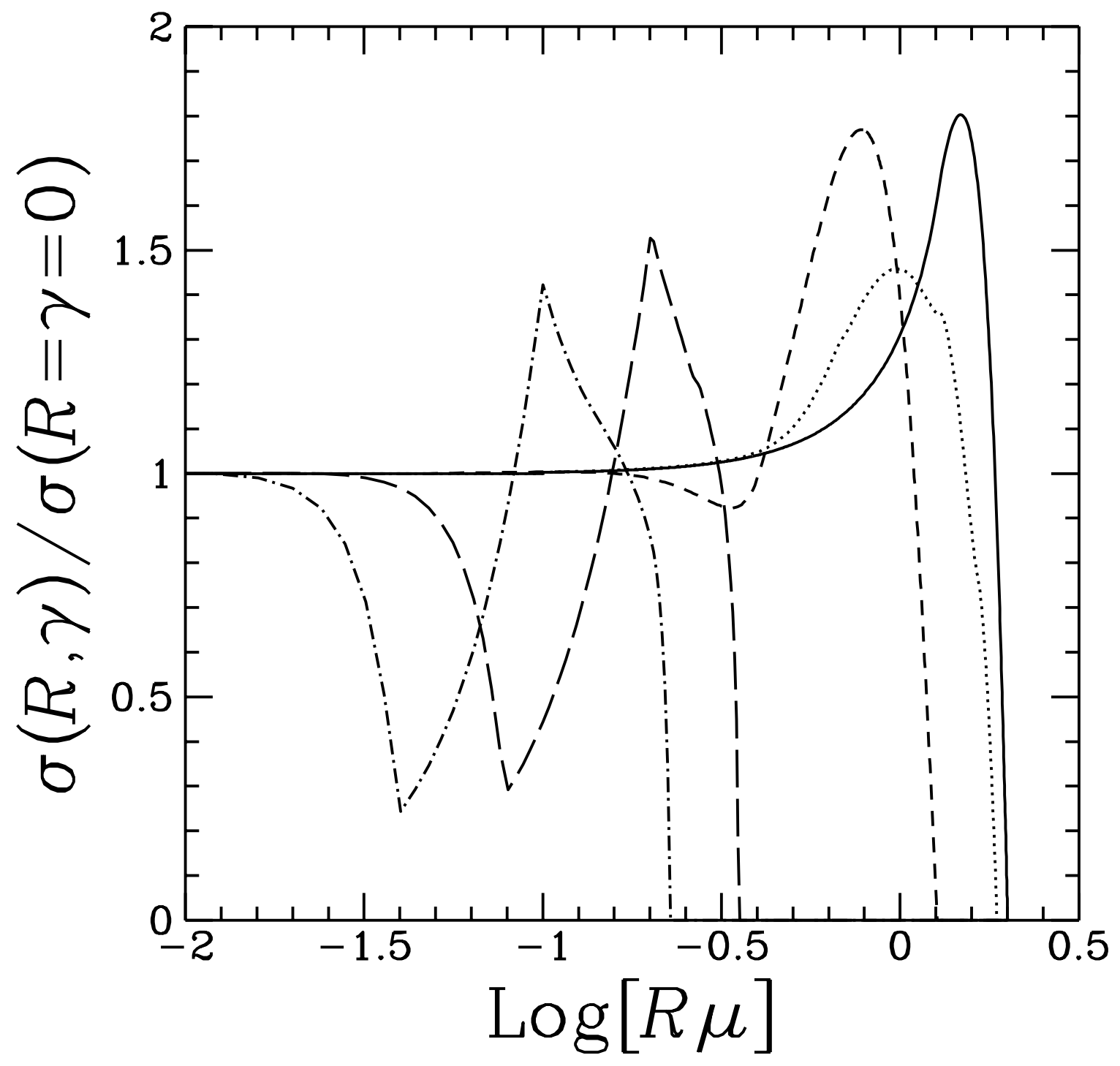

Fig. 2.- Cross-sections of magnification for lightcurves in Fig.1 (with the same line types). 


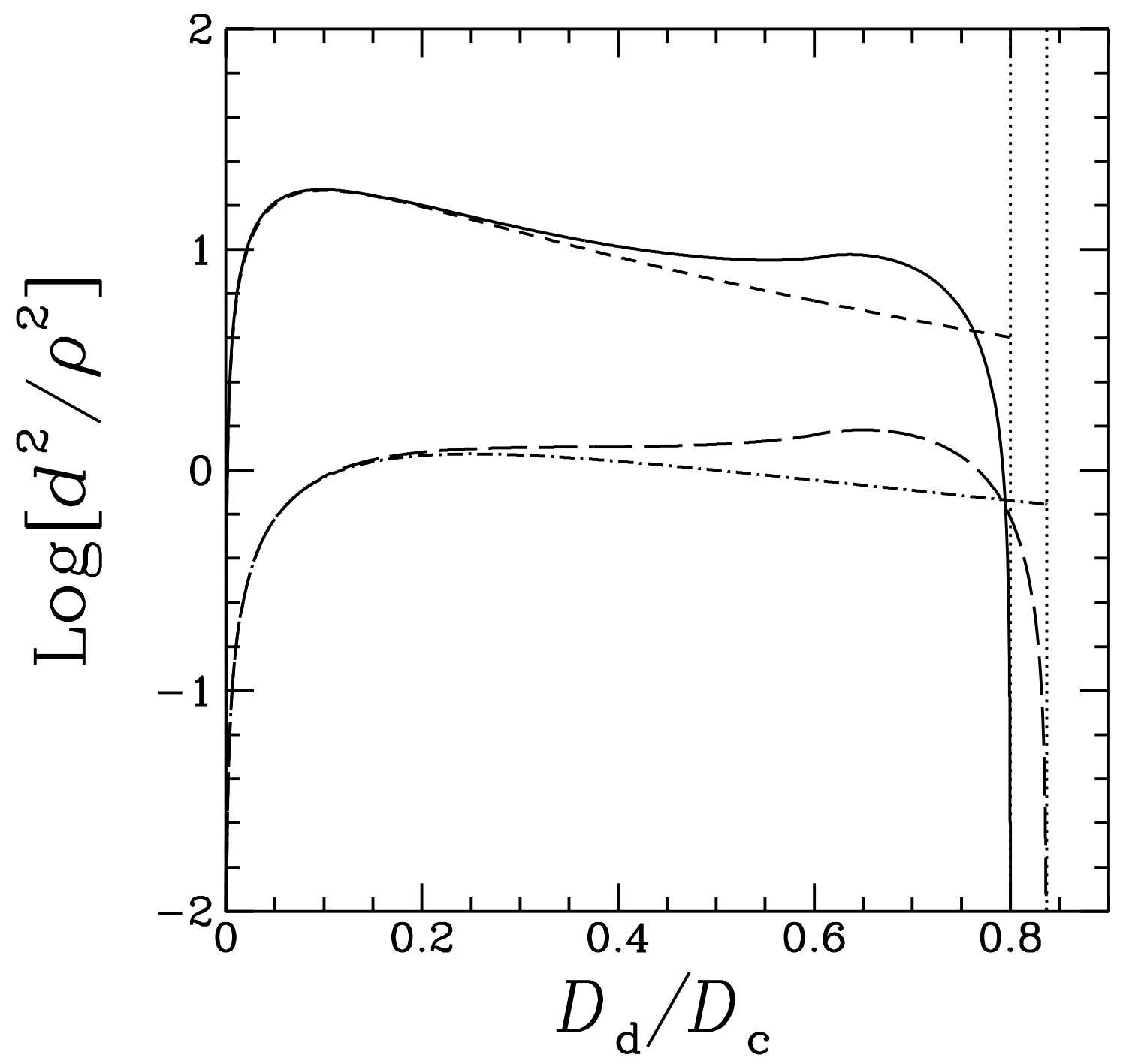

Fig. 3.- Cross-section $\sigma\left(f, \rho, D_{\mathrm{d}}\right)$ with $\alpha(f)=4$, for $D_{\mathrm{ds}}=0.2 D_{\mathrm{c}}$ (solid line), $0.5 D_{\mathrm{c}}$ (long dashed line). 


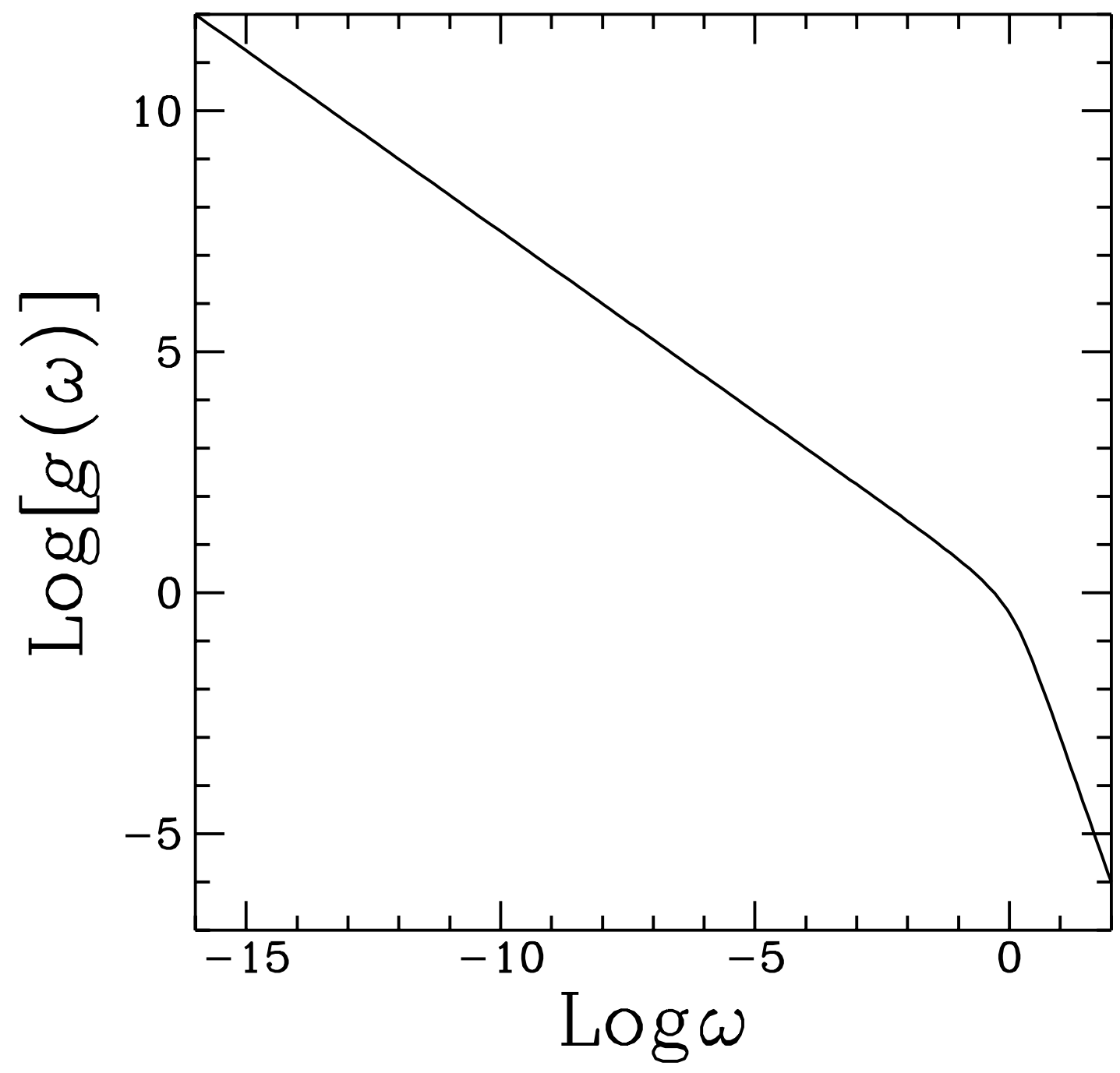

Fig. 4.- The scaled length of the high-flux $(>f)$ tube, $g(\omega)=D_{\mathrm{d}}^{\mathrm{m}}(f) / D_{\mathrm{ds}}$, as a function of $\omega=\left(D_{\mathrm{ds}} / D_{\mathrm{c}}\right) \alpha^{-1 / 3}(f)$. 


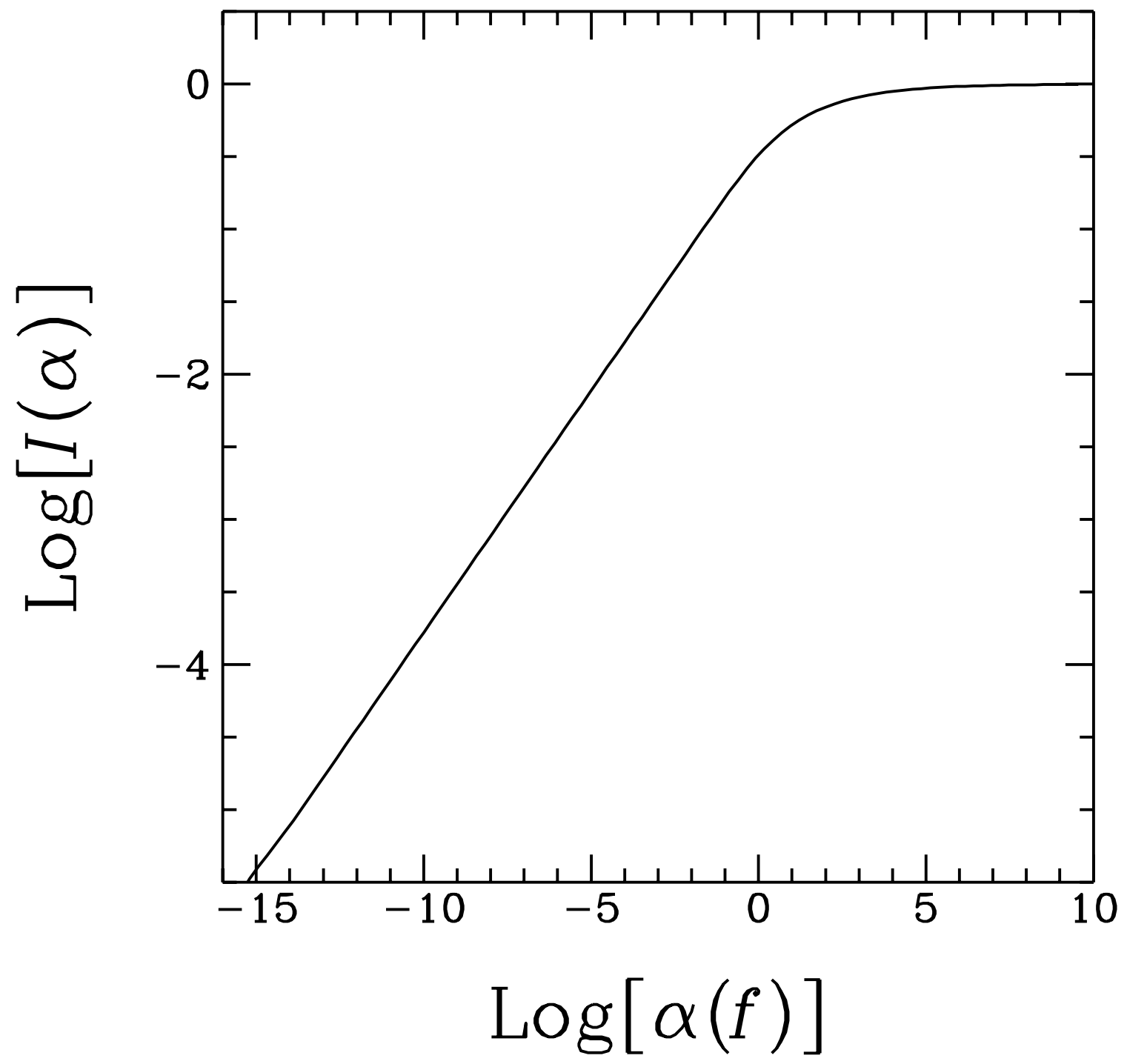

Fig. 5.- $I(\alpha)=V_{\mathrm{L}}(f, \rho) / V_{\mathrm{L}}(f, \rho=0)$, the reduction factor in EGLE volumes due to finite source size. 


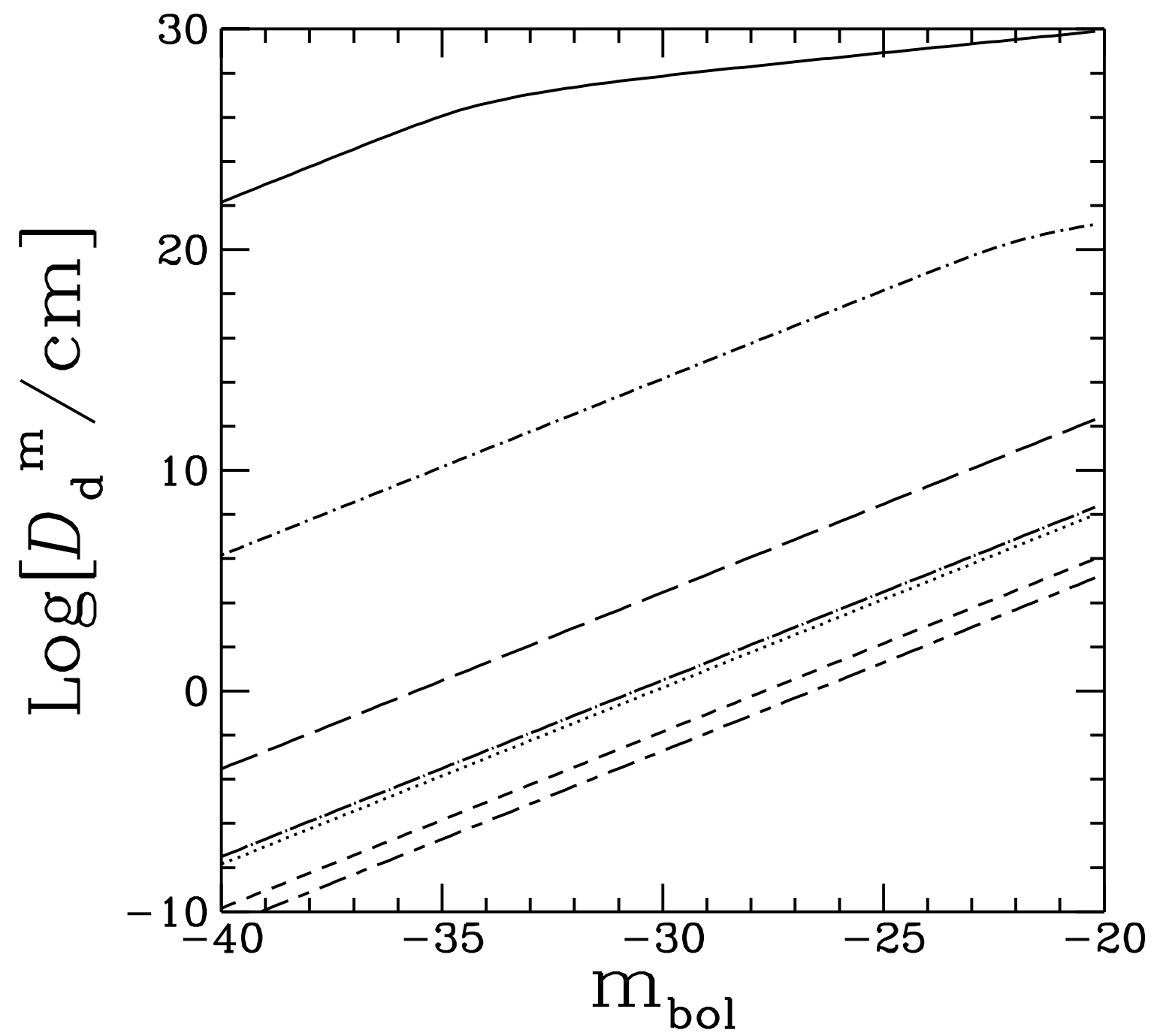

Fig. 6.- The high-flux $(>f)$ tube's length $D_{\mathrm{d}}^{\mathrm{m}}$ versus $m_{\text {bol }}(f)$ for various sources lensed by stars. The sources are $\gamma$-ray bursts (solid line), QSO (X-ray) (dotted line), QSO (UVopt) (short dashed line), Galactic supernovae (long dashed line), neutron stars (dot-short dashed line), hot O stars (dot-long dashed line), and hot B stars (short dash-long dashed line) respectively. 


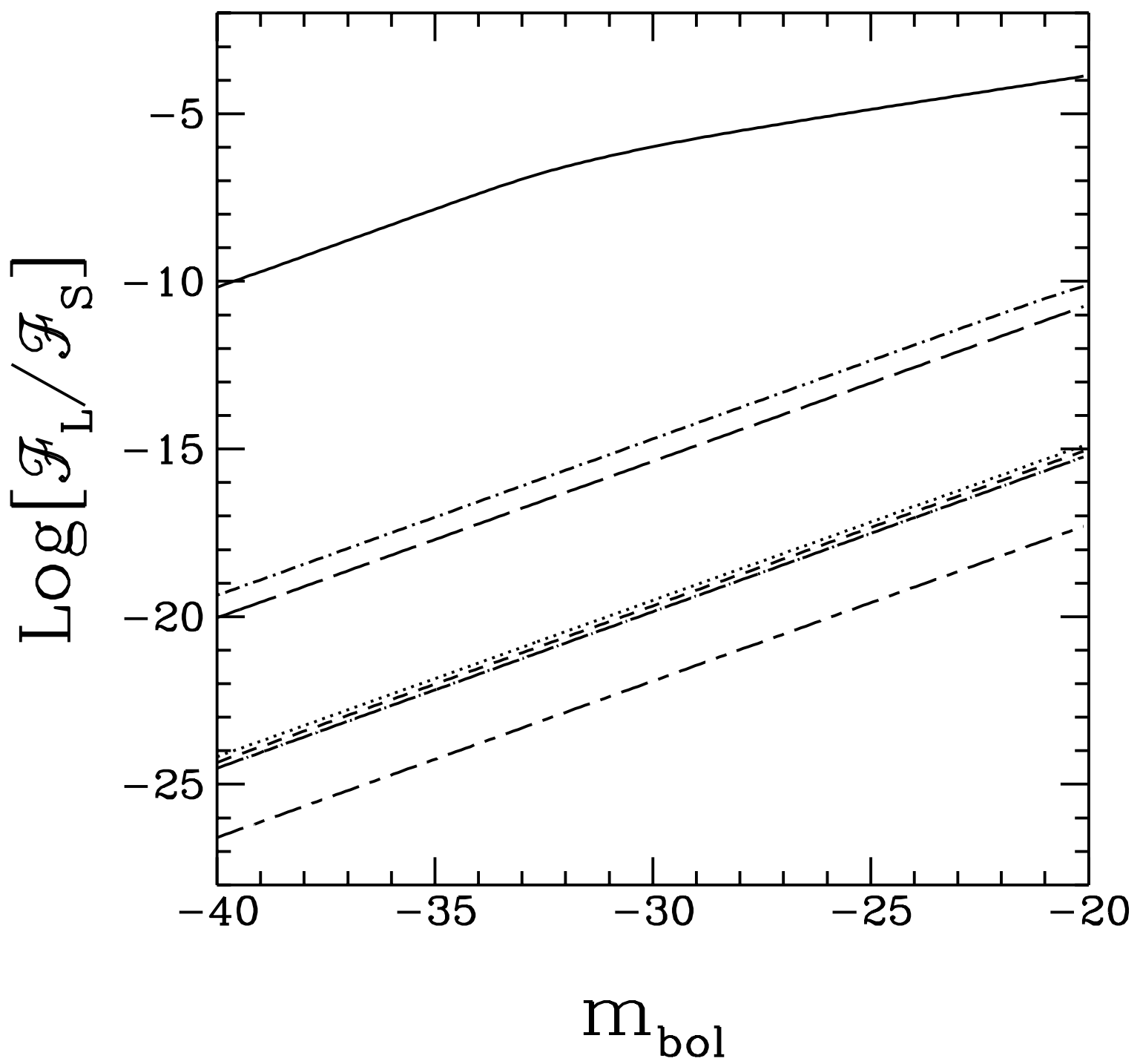

Fig. 7.- Lensing by stars of the same sources as in Fig.6 (with the same line types). (a) $\log \left(\mathcal{F}_{\mathrm{L}} / \mathcal{F}_{\mathrm{S}}\right)$ 


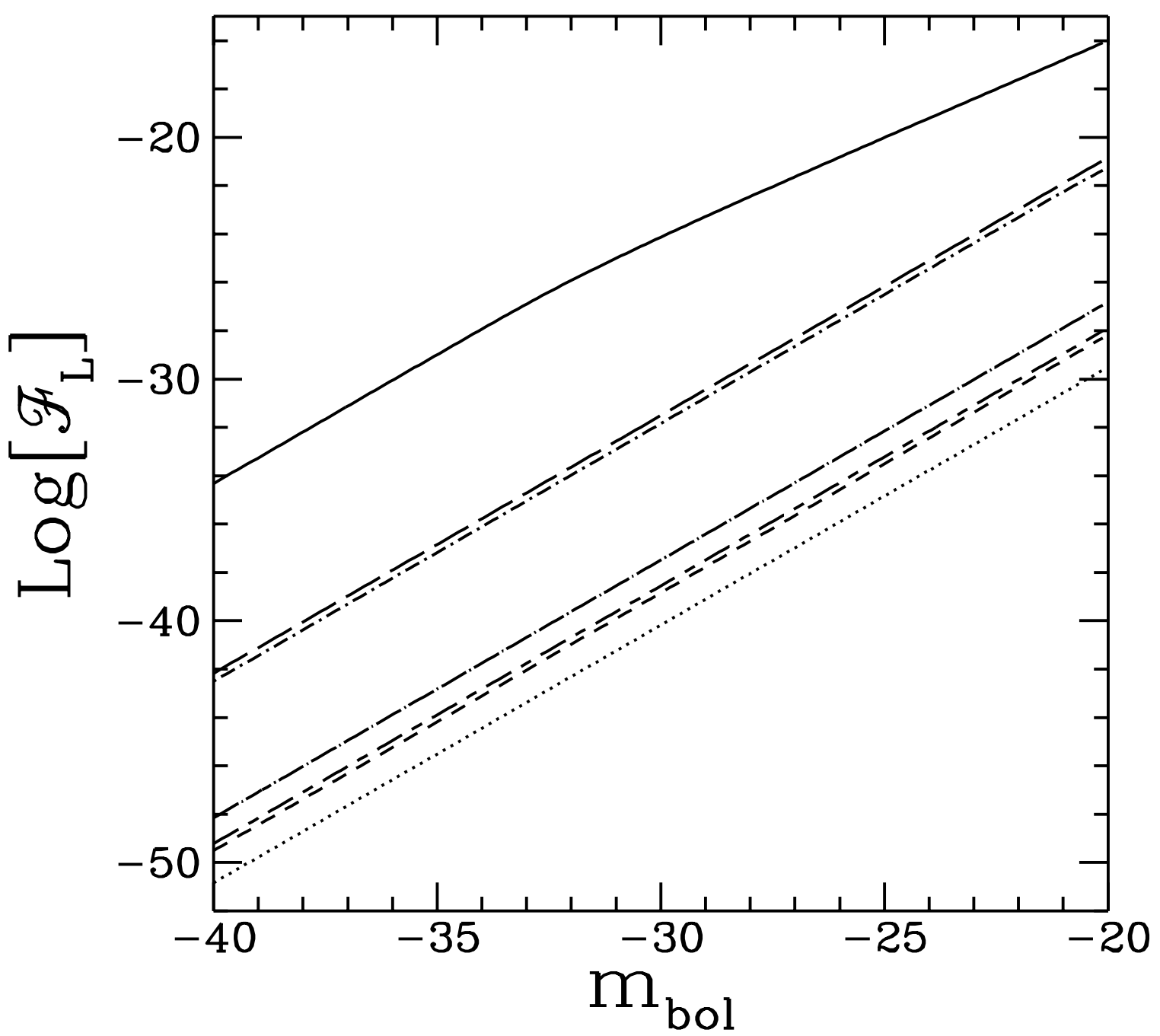

Fig. 7.- (b) $\log \mathcal{F}_{\mathrm{L}}$. 


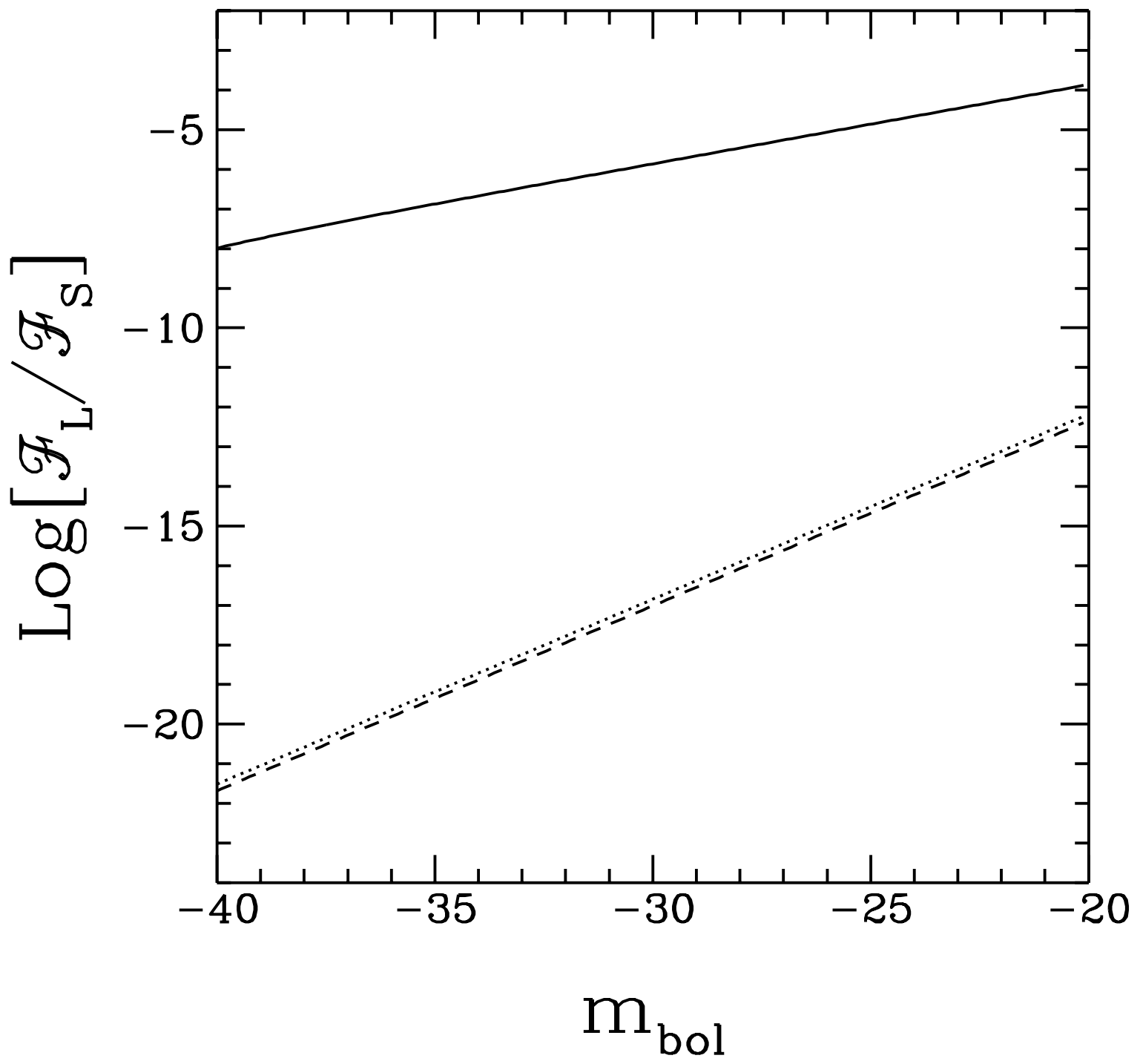

Fig. 8.- Lensing by giant black holes of the same cosmological sources as in Fig.6 (with the same line types). (a) $\log \left(\mathcal{F}_{\mathrm{L}} / \mathcal{F}_{\mathrm{S}}\right)$. 


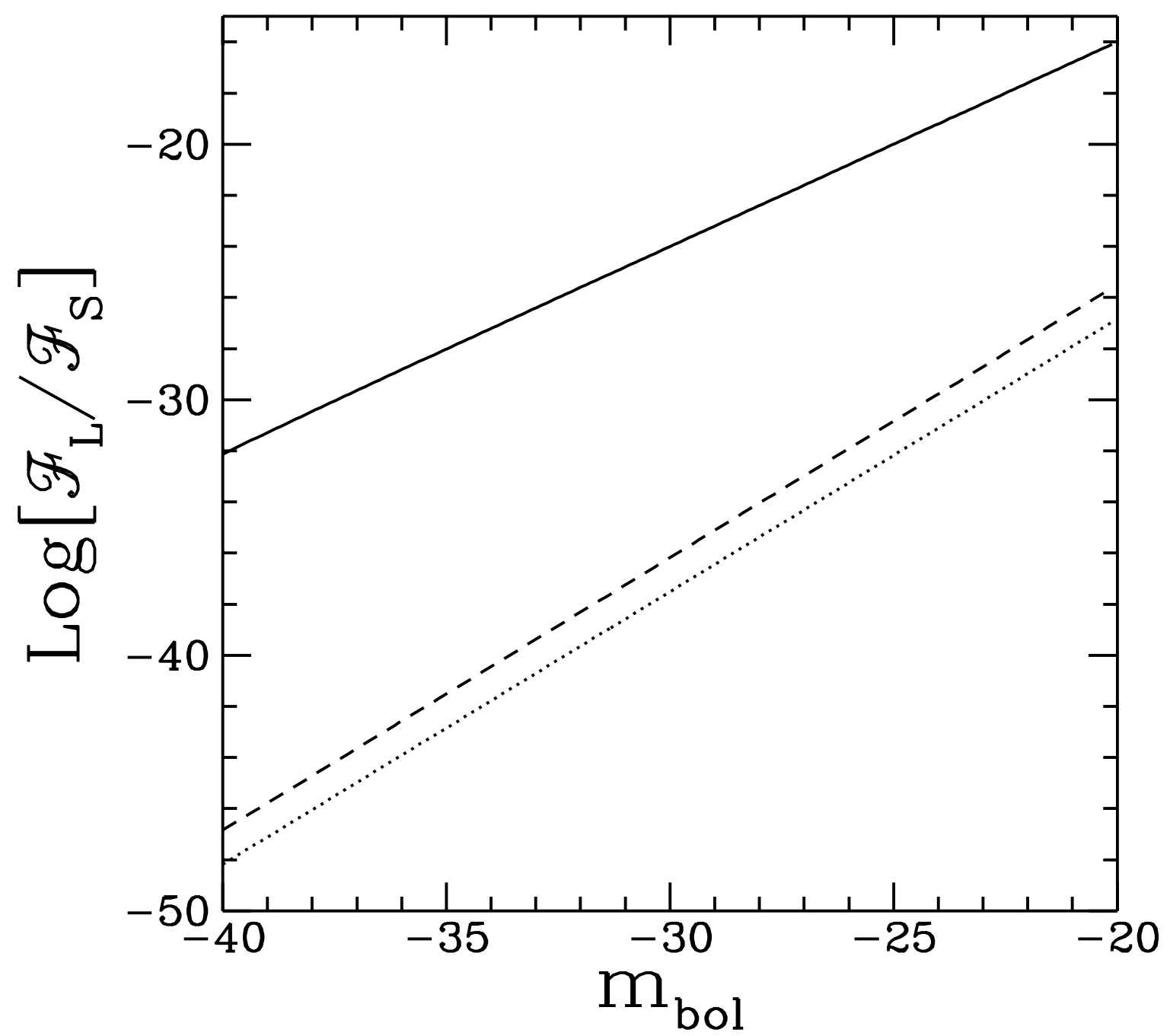

Fig. 8.- (b) $\log \left(\mathcal{F}_{\mathrm{L}}\right)$. 


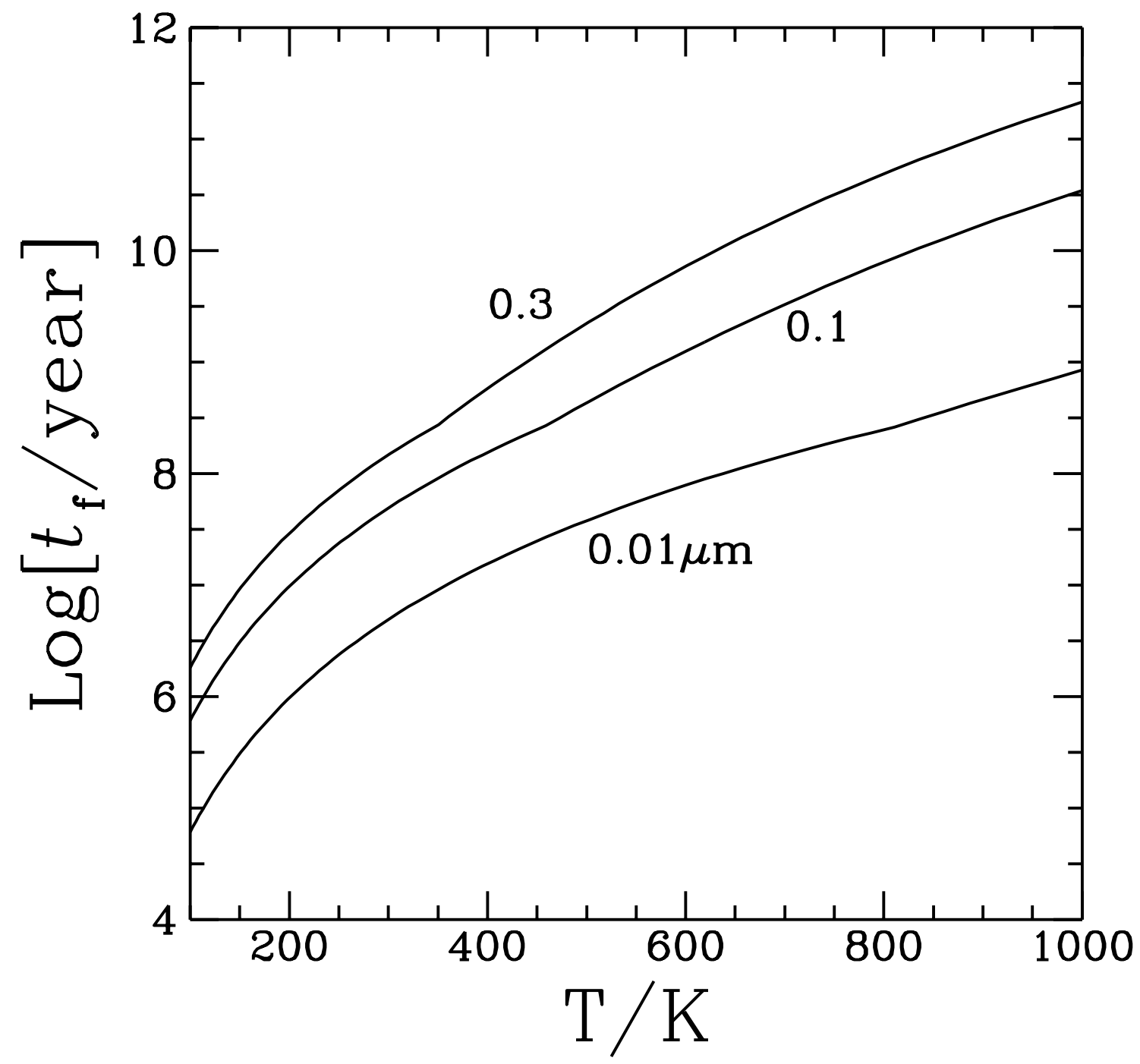

Fig. 9.- The time between two EGLEs as function of the silicate grain temperature, for X-ray/UV sources lensed by stars in a typical globular cluster. The values of the grain radius are labeled on the curves. 